\title{
Synthesis and Characterization of ( $\beta$-Diketonate)(7-tert-butoxynorbornadiene)copper(I) Compounds, a Series of New Copper CVD Precursors
}

\author{
Kai-Ming Chi,* Hung-Chang Hou, and Pao-Ts'un Hung \\ Department of Chemistry, National Chung Cheng University, Ming-Hsiung, Chia-Yi, \\ Taiwan, Republic of China \\ Shie-Ming Peng* and Gene-Hsiang Lee \\ Department of Chemistry, National Taiwan University, Taipei, Taiwan, Republic of China
}

Received November $21,1994^{\otimes}$

\begin{abstract}
Organocopper compounds of the general formula ( $\beta$-diketonate)Cu(7-t-BuO-NBD) where 7-t-BuO-NBD $=7$-tert-butoxynorbornadiene and $\beta$-diketonate $=1,1,1,5,5,5$-hexafluoro-2,4pentanedionate (1), 1,1,1-trifluoro-2,4-pentanedionate (2), 2,4-pentanedionate (3), 4,4,4trifluoro-1-(2-thienyl)-1,3-butanedionate (4), 4,4,4-trifluoro-1-phenyl-1,3-butanedionate (5), and 2,2-dimethyl-6,6,7,7,8,8,8-heptafluoro-3,5-octanedionate (6) were prepared by reaction of $\mathrm{CuCl}$ with $\mathrm{Na}(\beta$-diketonate) in the presence of $7-t-\mathrm{BuO}-\mathrm{NBD}$. All compounds were characterized by elemental analyses and ${ }^{1} \mathrm{H},{ }^{15} \mathrm{C}$, and ${ }^{19} \mathrm{~F}$ NMR, IR, and mass spectra. Singlecrystal structures of compounds 1, 4, and 5 were determined by X-ray diffraction analyses that showed mononuclear copper species with coordination of a chelating $\beta$-diketonate ligand through two oxygen atoms and the 7-t-BuO-NBD through one $\mathrm{C}=\mathrm{C}$ double bond and an oxygen atom in the solid state. Variable-temperature ${ }^{1} \mathrm{H}$ NMR spectral data of compound 2 in the solution are consistent with this structural nature. Hot-wall chemical vapor deposition experiments revealed that compound 1 is suitable as a precursor to deposit copper films in the temperature range $200-260^{\circ} \mathrm{C}$. Crystallographic data for compounds 1, 4, and 5: (hfac)Cu(7-t-BuO-NBD) (1) crystallizes in monoclinic system, space group $P 2 / 1, a=$ 8.923(1) $\AA, b=19.558(4) \AA, c=10.495(3) \AA, \beta=90.45(2)^{\circ}, V=1831.3(7) \AA^{3}, Z=4$. (Ttfac)$\mathrm{Cu}\left(7-t\right.$-BuO-NBD) (4) crystallizes in monoclinic system, space group $P 2_{1} / n, a=10.689(6) \AA$, $b=9.712(4) \AA, c=19.323(4) \AA, \beta=97.22(3)^{\circ}, V=1990.2(15) \AA^{3}, Z=4$. (Btfac)Cu(7-t BuO-NBD) (5) crystallizes in triclinic system, space group $P \overline{1}, a=9.617(1) \AA, b=9.894(4)$ $\AA, c=11.322(2) \AA, \alpha=104.57(2)^{\circ}, \beta=95.50(1)^{\circ}, \gamma=104.76(3)^{\circ}, V=993.4(5) \AA^{3}, Z=2$.
\end{abstract}

\section{Introduction}

Metal-organic chemical vapor deposition (MOCVD) of metals has a large number of important applications in the electronics and coatings industries. ${ }^{1}$ For instance, CVD of aluminum ${ }^{2}$ and tungsten ${ }^{3}$ thin films are commonly used to deposit interconnect layers in microelectronic devices. The use of copper(I) compounds as precursors of chemical vapor deposition of copper attracts much research interest as the result of potential applications in the microelectronic industry. ${ }^{4-8}$ Lewis base-stabilized ( $\beta$-diketonate)copper(I) complexes receive the most attention because they deposit highly

\footnotetext{
* Author to whom correspondence should be addressed.

* Abstract published in Advance ACS Abstracts, April 15, 1995.

(1) (a) Sherman, A. Chemical Vapor Deposition for Microelectronics: Principles, Technology, and Applications; Noyes Publications: Park Ridge, NJ, 1987. (b) Hess, D. W.; Jensen, K. F. Microelectronics Processing, Chemical Engineering Aspects; American Chemical Society: Washington, D. C., 1989.

(2) (a) Broadbent, E. K.; Ramiller, C. L. J. Electrochem. Soc. 1984 131, 1427. (b) Green, M. L.; Levy, R. A. J. Electrochem. Soc. 1985 132,1243

(3) (a) Levy, R. A.; Green, M. L.; Gallagher, R. K. J. Electrochem. Soc. 1984, 131, 2175. (b) Bent, B. E.; Nuzzo, R. G.; Dubois, L. H. J. Am. Chem. Soc. 1989, 111, 1634. (c) Beach, D. B.; Blum, S. E. LeGroues, F. K. J. Vac. Sci. Technol. 1989, A7, 3117. (d) Gross, M E.; Dubois, L. H.; Nuzzo, R. G.; Cheung, K. P. Mat. Res. Soc. Symp Proc. 1991, 204, 383. (e) Dubois, L. H.; Zegarski, B. R.; Gross, M. E.; Nuzzo, R. G. Surface Sci. 1991, 244, 89.
}

pure copper films via the disproportionation reaction of eq 1 . Although such complexes have been known for 2( $\beta$-diketonate $) \mathrm{Cu} \cdot \mathrm{L} \rightarrow$

$$
\mathrm{Cu}+\mathrm{Cu}(\beta \text {-diketonate })_{2}+2 \mathrm{~L}
$$

about 30 years, ${ }^{9}$ relatively little structural information is available. ${ }^{8 \mathrm{~b}, 10-12}$ Therefore, it is valuable to systematically investigate the structural properties of these compounds to provide insight into their volatility,

(4) $\left(\eta^{5}-\mathrm{C}_{5} \mathrm{H}_{5}\right) \mathrm{Cu}\left(\mathrm{PR}_{3}\right)$ as MOVCD precursors: (a) Dupuy, C. G.; Beach, D. B.; Hurst, J. E. Jr.; Jasinki, J. M. Chem. Mater. 1989, 1, 16. (b) Beach, D. B.; LeGroues, F. A.; Hu, C.-K. Chem. Mater. 1990, 2 , 216. (c) Hampden-Smith, M. J.; Kodas, T. T.; Paffett, M.; Farr, J. D.; Shin, H. K. Chem. Mater. 1990, 2, 636.

(5) $(t-\mathrm{BuOCu})_{4}$ as MOCVD precursor: (a) Jeffries, P. M.; Girolami, G. S. Chem. Mater. 1989, 1,8. (b) Jeffries, P. M.; Dubois, L. H.; Girolami, G. S. Chem. Mater. $1992,4,1169$

(6) $\left(\beta\right.$-diketonate) $\mathrm{Cu}\left(\mathrm{PR}_{3}\right)$ as MOCVD precursors: (a) Shin, H.-K. Chi, K.-M.; Hampden-Smith, M. J.; Kodas, T. T.; Farr, J. D.; Paffett, M. Adv. Mater. 1991, 3, 246. (b) Shin, H. K.; Chi, K. M.; HampdenSmith, M. J.; Kodas, T. T.; Farr, J. D.; Paffett, M. Chem. Mater, 1992 $4,788$.

(7) ( $\beta$-diketonate)Cu(alkene) as MOCVD precursors: (a) Reynolds, S. K.; Smart, C. J.; Baran, E. F.; Baum, T. H.; Larson, C. E.; Brock, P J. Appl. Phys. Lett. 1991, 59, 2332. (b) Jain, A.; Chi, K.-M.; HampdenSmith, M. J.; Kodas, T. T.; Paffett, M.; Farr, J. D. J. Mater. Res, 1992 7, 261. (c) Norman, J. A. T.; Muratore, B. A.; Dyer, P. N.; Robert, D. A.; Hochberg, A. K. J. Phys. (Paris) 1991, IV, C2/271. (d) Jain, A.; Chi, K.-M.; Hampden-Smith, M. J.; Kodas, T. T. J. Electrochem. Soc. 1993, 140, 1434.

(C) 1995 American Chemical Society 
stability, and reactivity, especially in relation with CVD. Here, we report the syntheses and characterizations of a series of new ( $\beta$-diketonate)Cu(alkene) complexes with coordination number 4 , in which alkene $=7$-tert butoxynorbornadiene (7-t-BuO-NBD) and $\beta$-diketonate $=1,1,1,5,5,5$-hexafluoro-2,4-pentanedionate $(\mathrm{hfac})(1)$, 1,1,1-trifluoro-2,4-pentanedionate (tfac) (2), 2,4-pentanedionate (acac) (3), 4,4,4-trifluoro-1-(2-thienyl)-1,3butanedionate (Ttfac) (4), 4,4,4-trifluoro-1-phenyl-1,3butanedionate (Btfac) (5), and 2,2-dimethyl-6,6,7,7,8,8,8heptafluoro-3,5-octanedionate (fod) (6), variable-temperature ${ }^{1} \mathrm{H}$ NMR spectroscopic studies of the title compounds, single-crystal structures of compounds 1 , 4, and 5, and the results of hot-wall CVD experiments with compound 1 as a precursor.

\section{Experimental Section}

General Procedures and Starting Materials. All operations were performed under an atmosphere of nitrogen purified by passage through columns of activated BASF catalyst and molecular sieves and using standard Schlenk techniques ${ }^{13}$ in conjunction with a double manifold vacuum line. All hydrocarbon and ethereal solvents were dried and distilled from sodium benzophenone ketyl at atmospheric pressure before use. Copper(I) chloride, sodium hydride, 1,1,1,5,5,5-hexafluoro-2,4-pentanedione, 1,1,1-trifluoro-2,4pentanedione, 2,4-pentanedione, 4,4,4-trifluoro-1-(2-thienyl)1,3-butanedione, 4,4,4-trifluoro-1-phenyl-1,3-butanedione, and 2,2-dimethyl-6,6,7,7,8,8,8-heptafluoro-3,5-octanedione (Aldrich Chemical Co.) were used without further purification. The sodium salts of the $\beta$-diketones ${ }^{10 \mathrm{~b}}$ and the diene 7 -tertbutoxynorbornadiene ${ }^{14}$ were prepared by the methods previously described in the literature. Elemental analyses and mass spectral analyses were made at National Science Council Southern Instrument Center (Department of Chemistry, National Cheng Kung University). NMR data were recorded on a Varian Gemini-200 NMR spectrometer by using the protio impurities of deuterated solvents as references for the ${ }^{1} \mathrm{H}$ NMR and the ${ }^{13} \mathrm{C}$ signals of the solvents as references for ${ }^{13} \mathrm{C}$ NMR spectroscopies. ${ }^{19} \mathrm{~F}$ NMR spectra were externally referred to $\mathrm{CFCl}_{3}$. Infrared data were recorded on a Perkin-Elmer Model 16 PC FTIR spectrophotometer. Melting points were measured in sealed capillaries on a Thomas-Hoover Unimelt instrument without calibration.

Synthesis and Characterization of ( $\beta$-Diketonate) $\mathrm{Cu}$ (7- $t$-BuO-NBD). a. (hfac)Cu(7-t-BuO-NBD) (1). Addition of $7-t$-BuO-NBD $(6.2 \mathrm{~mL}, 32.3 \mathrm{mmol})$ to a $250-\mathrm{mL}$ Schlenk flask containing a solution of $\mathrm{CuCl}(3.21 \mathrm{~g}, 32.3 \mathrm{mmol})$ in diethyl ether $(50 \mathrm{~mL})$ gave a white slurry solution. A solution of $\mathrm{Na}$ (hfac) $(7.52 \mathrm{~g}, 32.7 \mathrm{mmol})$ in $\mathrm{Et}_{2} \mathrm{O}(50 \mathrm{~mL})$ was transferred into the reaction flask with stirring, and the reaction mixture turned yellow immediately. The mixture was stirred for $4 \mathrm{~h}$

(8) ( $\beta$-diketonate)Cu(alkyne) as MOCVD precursors: (a) Jain, A Chi, K.-M.; Kodas, T. T.; Hampden-Smith, M. J.; Farr, J. D.; Paffett, M. Chem. Mater. 1991, 3, 995. (b) Baum, T. H.; Larson, C. E. Chem. Mater. 1992, 4, 365.

(9) Nast, R.; Lepel, W.-H. Chem. Ber. 1969, 102, 3224

(10) (a) Anderson, W. A.; Carty, A. J.; Palenik, G. J.; Schreiber, G. Can. J. Chem. 1971, 49, 761. (b) Shin, H.-K.; Chi, K. M.; Farkas, J. Hampden-Smith, M. J.; Kodas, T. T.; Duesler, E. N. Inorg. Chem. 1992, 31, 424. (c) Chi, K. M.; Corbitt, T. S.; Hampden-Smith, M. J.; Kodas, T. T.: Duesler, E. N. J. Organomet. Chem, 1993, 449, 181.

(11) (a) Doyle, G.; Eriksen, K. A.; Van Engen, D. Organometallics 1985, 4, 830. (b) Chi, K. M.; Shin, H.-K.; Hampden-Smith, M. J. Kodas, T. T.; Duesler, E. N. Polyhedron 1991, 10, 2293. (c) Kumar, R.; Fronczek, F. R.; Mavreick, A. W.; Lai, W. G.; Griffin, G. F. Chem. Mater. 1992, 4, 577 .

(12) Chi, K. M.; Shin, H.-K.; Hampden-Smith, M. J.; Kodas, T. T.; Duesler, E. N. Inorg. Chem, 1991, 30, 424.

(13) Shriver, D. F;; Drezden, M. A. The Manipulation of Air-Sensitive Compounds, 2nd ed.; Wiley: New York, 1986.

(14) Story, P. R.; Fahrenholtz, S. R. Organic Syntheses; Wiley: New York, 1973; Collect. Vol. V, p 151. at room temperature. The volatile components were removed in vacuo $(0.1$ Torr $)$, and hexane $(80 \mathrm{~mL})$ was added to dissolve the product. After filtration and removal of hexane, a yellow powder was obtained. Recrystallization of the compound from hexane $(10 \mathrm{~mL})$ at $0{ }^{\circ} \mathrm{C}$ afforded yellow, crystalline (hfac) Cu(7-t-BuO-NBD) $(10.2 \mathrm{~g}, 73 \%$ yield $)$.

Anal. Calcd for $\mathrm{C}_{16} \mathrm{H}_{17} \mathrm{O}_{3} \mathrm{~F}_{6} \mathrm{Cu}: \mathrm{C}, 44.19 ; \mathrm{H}, 3.94$. Found: C, 44.31; H, 3.93.

NMR data $\left(\mathrm{C}_{6} \mathrm{D}_{6}, 18^{\circ} \mathrm{C}\right)$ : ${ }^{1} \mathrm{H} \delta 6.27$ (s, $1 \mathrm{H}, \mathrm{CH}$ on hfac), $6.04(\mathrm{~m}, 2 \mathrm{H}$, noncoordinated $\mathrm{CH}=\mathrm{CH}$ on $7-t-\mathrm{BuO}-\mathrm{NBD}), 5.02$ (br, s, $2 \mathrm{H}$, coordinated $\mathrm{CH}=\mathrm{CH}$ on $7-t-\mathrm{BuO}-\mathrm{NBD}$ ), 3.31 (br, s, $1 \mathrm{H}$, bridge $H$ on $7-t-\mathrm{BuO}-\mathrm{NBD}$ ), 2.93 (br, s, 2H, bridgehead $H$ on 7-t-BuO-NBD), 0.85 (s, $9 \mathrm{H}, \mathrm{C}\left(\mathrm{CH}_{3}\right)_{3}$ on 7-t-BuO-NBD) ppm; ${ }^{13} \mathrm{C}\left\{{ }^{1} \mathrm{H}\right\} \delta 178.3\left(\mathrm{q}, J_{\mathrm{FC}}=33.7 \mathrm{~Hz}, \mathrm{CF}_{3} \mathrm{CO}\right.$ on hfac), $139.6(\mathrm{~s}$, noncoordinated $\mathrm{CH}=\mathrm{CH}$ on $7-t-\mathrm{BuO}-\mathrm{NBD}$ ), 119.3 (q, $J_{\mathrm{FC}}=$ $284.5 \mathrm{~Hz}, C \mathrm{~F}_{3}$ on hfac), 101.9 (s, coordinated $\mathrm{CH}=\mathrm{CH}$ on $7-t$ BuO-NBD), 92.7 (s, bridge $C$ on 7-t-BuO-NBD), 90.1 (s, $C H$ on hfac), $76.8\left(\mathrm{~s},\left(\mathrm{CH}_{3}\right)_{3} \mathrm{C}\right.$ on $7 \cdot t-\mathrm{BuO}-\mathrm{NBD}$ ), 56.0 (s, bridgehead $C$ on $7-t-\mathrm{BuO}-\mathrm{NBD}), 28.2\left(\mathrm{~s},\left(\mathrm{CH}_{3}\right)_{3} \mathrm{C}\right.$ on $\left.7-t-\mathrm{BuO}-\mathrm{NBD}\right) \mathrm{ppm}$; ${ }^{19} \mathrm{~F}\left\{{ }^{1} \mathrm{H}\right\} \delta-76.3(\mathrm{~s}) \mathrm{ppm}$.

IR data (KBr disk): $3764(\mathrm{w}), 2986(\mathrm{w}), 1644(\mathrm{~s}), 1574(\mathrm{~m})$, $1540(\mathrm{~m}), 1488(\mathrm{~s}), 1387(\mathrm{~m}), 1262(\mathrm{~s}), 1194(\mathrm{~s}), 1144(\mathrm{~s}), 1084$ (s), $898(\mathrm{w}), 800(\mathrm{~m}), 676(\mathrm{~m}), 586(\mathrm{w}) \mathrm{cm}^{-1}$.

Mass spectral data $\left(\mathrm{FAB}^{+}, \mathrm{m} / z\right): 434,34.5[\mathrm{Cu}(\mathrm{hfac})(7-t$ BuO-NBD) $]^{++} ; 378,81.8[\mathrm{Cu}(\mathrm{hfac})(7-\mathrm{HO}-\mathrm{NBD})]^{0+} ; 227,39.4$ $\left[\mathrm{Cu}(7-t \text {-BuO-NBD) }]^{++} ; 171,23.8[\mathrm{Cu}(7-\mathrm{HO}-\mathrm{NBD})]^{0+} ; 139,100\right.$ $\left[\mathrm{CF}_{3} \mathrm{C}(\mathrm{O}) \mathrm{CHC}(\mathrm{O}) \mathrm{H}\right]^{++} ; 107,50.2[(7-\mathrm{O}-\mathrm{NBD})]^{++} ; 91,62.7\left[\mathrm{C}_{7} \mathrm{H}_{7}\right]^{]^{+}}$; $77,47.5\left[\mathrm{C}_{6} \mathrm{H}_{5}\right]^{++} ; 63,17.9[\mathrm{Cu}]^{++} ; 57,50.3\left[\mathrm{C}_{4} \mathrm{H}_{9}\right]^{++}$

Mp: $105^{\circ} \mathrm{C}$, sublimation temperature: $75^{\circ} \mathrm{C} / 0.1$ Torr.

b. (tfac)Cu(7-t-BuO-NBD) (2) Pale yellow, crystalline (tfac) $\mathrm{Cu}(7-t-\mathrm{BuO}-\mathrm{NBD})$ was prepared in $60 \%$ yield with a procedure analogous to that of synthesis of compound 1 .

Anal. Calcd for $\mathrm{C}_{16} \mathrm{H}_{20} \mathrm{O}_{3} \mathrm{~F}_{3} \mathrm{Cu}: \mathrm{C}, 50.46 ; \mathrm{H}, 5.29$. Found: C, 50.16; H, 5.28.

NMR data $\left(\mathrm{C}_{6} \mathrm{D}_{6}, 18^{\circ} \mathrm{C}\right):{ }^{1} \mathrm{H} \delta 6.09(\mathrm{~m}, 2 \mathrm{H}$, noncoordinated $\mathrm{CH}=\mathrm{CH}$ on 7-t-BuO-NBD), $5.77(\mathrm{~s}, 1 \mathrm{H}, \mathrm{CH}$ on tfac), $4.96(\mathrm{br}$, $\mathrm{s}, 2 \mathrm{H}$, coordinated $\mathrm{CH}=\mathrm{CH}$ on 7-t-BuO-NBD), $3.40(\mathrm{br}, \mathrm{s}, 1 \mathrm{H}$, bridge $H$ on $7-t-\mathrm{BuO}-\mathrm{NBD}$ ), 3.05 (br, s, $2 \mathrm{H}$, bridgehead $H$ on 7-t-BuO-NBD), $1.75\left(\mathrm{~s}, 3 \mathrm{H}, \mathrm{CH}_{3}\right.$ on tfac), $0.99\left(\mathrm{~s}, 9 \mathrm{H}, \mathrm{C}\left(\mathrm{CH}_{3}\right)_{3}\right.$ on 7-t-BuO-NBD) ppm; ${ }^{13} \mathrm{C}\left\{{ }^{1} \mathrm{H}\right\} \delta 197.9$ (s, $\mathrm{CH}_{3} \mathrm{CO}$ on tfac), $171.4\left(\mathrm{q}, J_{\mathrm{FC}}=31.0 \mathrm{~Hz}, \mathrm{CF}_{3} \mathrm{CO}\right.$ on tfac), 139.8 (s, noncoordinated $C H=C H$ on $7-t-B u O-N B D), 120.8\left(q, J_{\mathrm{FC}}=284.1 \mathrm{~Hz}\right.$, $\mathrm{CF}_{3}$ on tfac), 97.4 (s, coordinated $\mathrm{CH}=\mathrm{CH}$ on 7-t-BuO-NBD), 95.3 (s, $C \mathrm{H}$ on tfac), 92.2 (s, bridge $C$ on $7-t-\mathrm{BuO}-\mathrm{NBD}$ ), 76.5 $\left(\mathrm{s},\left(\mathrm{CH}_{3}\right)_{3} \mathrm{C}\right.$ on $\left.7-t-\mathrm{BuO}-\mathrm{NBD}\right), 55.9$ (s, bridgehead $C$ on $7-t-$ BuO-NBD), $29.6\left(\mathrm{~s}, \mathrm{CH}_{3}\right.$ on tfac), $28.5\left(\mathrm{~s},\left(\mathrm{CH}_{3}\right)_{3} \mathrm{C}\right.$ on $7-t-\mathrm{BuO}-$ NBD) ppm; ${ }^{19} \mathrm{~F}\left\{{ }^{1} \mathrm{H}\right\} \delta-75.4(\mathrm{~s}) \mathrm{ppm}$.

IR data ( $\mathrm{KBr}$ disc): $3764(\mathrm{w}), 2980(\mathrm{w}), 1628$ (s), 1518 (s), $1497(\mathrm{~m}), 1360(\mathrm{w}), 1298(\mathrm{~s}), 1233(\mathrm{~m}), 1182(\mathrm{~s}), 1136(\mathrm{~s}), 1084$ (s), $886(\mathrm{w}), 774(\mathrm{w}), 700(\mathrm{w}), 582(\mathrm{w}) \mathrm{cm}^{-1}$.

Mass spectral data $\left(\mathrm{FAB}^{+}, \mathrm{m} / z\right): 380,51.2[\mathrm{Cu}(\mathrm{tfac})(7-t-\mathrm{BuO}-$ NBD) $]^{++} ; 324,83.8[\mathrm{Cu}(\mathrm{tfac})(7-\mathrm{HO}-\mathrm{NBD})]^{++} ; 227,83.6[\mathrm{Cu}(7-$ $t$-BuO-NBD) $]^{++} ; 216,12.1[\mathrm{Cu}(\mathrm{tfac})]^{+} ; 171,50.5[\mathrm{Cu}(7-\mathrm{HO}-$ $\mathrm{NBD})]^{0+} ; 141,23.0\left[\mathrm{Cu}\left(\mathrm{C}_{6} \mathrm{H}_{6}\right)\right]^{0+} ; 107,22.0[(7-\mathrm{O}-\mathrm{NBD})]^{++} ; 92$, $39.3[\mathrm{NBD}]^{++} ; 91,100\left[\mathrm{C}_{7} \mathrm{H}_{7}\right]^{++} ; 79,10.7\left[\mathrm{C}_{6} \mathrm{H}_{7}\right]^{++} ; 63,15.2[\mathrm{Cu}]^{++}$ $57,51.2\left[\mathrm{C}_{4} \mathrm{H}_{9}\right]^{++}$.

$\mathrm{Mp}: 110^{\circ} \mathrm{C}$ dec, sublimation temperature: $75^{\circ} \mathrm{C} / 0.1$ Torr.

c. (acac)Cu(7-t-BuO-NBD) (3). White, crystalline (acac)$\mathrm{Cu}(7-t-\mathrm{BuO}-\mathrm{NBD})$ was prepared in $47 \%$ yield following a procedure similar to that of synthesis of compound 1 except that THF was used as the solvent for reaction.

Anal. Calcd for $\mathrm{C}_{16} \mathrm{H}_{23} \mathrm{O}_{3} \mathrm{Cu}: \mathrm{C}, 58.79 ; \mathrm{H}, 7.09$. Found: $\mathrm{C}$, $58.40 ; \mathrm{H}, 7.14$.

NMR data $\left(\mathrm{C}_{6} \mathrm{D}_{6}, 18{ }^{\circ} \mathrm{C}\right):{ }^{1} \mathrm{H} \delta 6.14(\mathrm{br}, \mathrm{s}, 2 \mathrm{H}$, noncoordinated $\mathrm{CH}=\mathrm{CH}$ on 7-t-BuO-NBD), 5.36 (s, $1 \mathrm{H}, \mathrm{CH}$ on acac), $4.88(\mathrm{br}, \mathrm{s}, 2 \mathrm{H}$, coordinated $\mathrm{CH}=\mathrm{CH}$ on $7-t-\mathrm{BuO}-\mathrm{NBD}), 3.47$ (br, s, $1 \mathrm{H}$, bridge $H$ on $7-t$-BuO-NBD), 3.16 (br, s, $2 \mathrm{H}$, bridgehead $H$ on $7-t-\mathrm{BuO}-\mathrm{NBD}), 1.94$ (s, $6 \mathrm{H}, \mathrm{CH}_{3}$ on acac), 1.09 (s, 9H, C $\left(\mathrm{CH}_{3}\right)_{3}$ on $\left.7-t-\mathrm{BuO}-\mathrm{NBD}\right) \mathrm{ppm} ;{ }^{13} \mathrm{C}\left\{{ }^{1} \mathrm{H}\right\} \delta 190.3(\mathrm{~s}, \mathrm{CO}$ on acac), 139.9 (s, noncoordinated $\mathrm{CH}=\mathrm{CH}$ on $7-t-\mathrm{BuO}-\mathrm{NBD}$ ), 100.0 (s, $C H$ on acac), 93.3 (s, coordinated $\mathrm{CH}=\mathrm{CH}$ on $7-t$-BuO$\mathrm{NBD}$ ), 91.6 (s, bridge $C$ on $7-t-\mathrm{BuO}-\mathrm{NBD}), 76.1\left(\mathrm{~s},\left(\mathrm{CH}_{3}\right)_{3} \mathrm{C}\right.$ on 
7-t-BuO-NBD), 55.8 (s, bridgehead $C$ on 7-t-BuO-NBD), 28.9

(s, $\mathrm{CH}_{3}$ on acac), 28.7 (s, $\left(\mathrm{CH}_{3}\right)_{3} \mathrm{C}$ on $\left.7-t-\mathrm{BuO}-\mathrm{NBD}\right) \mathrm{ppm}$.

IR data (KBr disk): $3083(\mathrm{w}), 2976(\mathrm{w}), 1596(\mathrm{~s}), 1514$ (s), $1462(\mathrm{~m}), 1406(\mathrm{~s}), 1363(\mathrm{~m}), 1320(\mathrm{w}), 1272(\mathrm{w}), 1232(\mathrm{~m})$, $1188(\mathrm{~m}), 1090(\mathrm{~s}), 1016(\mathrm{~m}), 936(\mathrm{w}), 890(\mathrm{w}), 672(\mathrm{~m}), 702$ $(\mathrm{m}), 572(\mathrm{w}) \mathrm{cm}^{-1}$

Mass spectral data $\left(\mathrm{EI}^{+}, m / z\right): 326,10.7[\mathrm{Cu}(\mathrm{acac})(7-t-\mathrm{BuO}$ NBD) $]^{++} ; 270,7.4[\mathrm{Cu}(\mathrm{acac})(7-\mathrm{HO}-\mathrm{NBD})]^{++} ; 261,10.3[\mathrm{Cu}-$ $\left.(\text { acac })_{2}\right]^{++} ; 163,13.0[\mathrm{HCu}(\mathrm{acac})]^{++} ; 147,9.1\left[\mathrm{Cu}\left(\mathrm{C}_{4} \mathrm{H}_{4} \mathrm{O}_{2}\right)\right]^{++} ; 108$, $31.1[(7-\mathrm{HO}-\mathrm{NBD})]^{0+} ; 107,18.8[(7-\mathrm{O}-\mathrm{NBD})]^{0+} ; 91,79.0\left[\mathrm{C}_{7} \mathrm{H}_{7}\right]^{0+}$; 85, $15.9\left[\left(\mathrm{C}_{4} \mathrm{H}_{4} \mathrm{O}_{2}\right)\right]^{*+} ; 79,50.9\left[\mathrm{C}_{6} \mathrm{H}_{7}\right]^{++} ; 78,19.6\left[\mathrm{C}_{6} \mathrm{H}_{6}\right]^{++} ; 63$, $6.3[\mathrm{Cu}]^{++} ; 57,100\left[\mathrm{C}_{4} \mathrm{H}_{9}\right]^{]^{++}}$

$\mathrm{Mp}: 115^{\circ} \mathrm{C}$ dec, sublimation temperature: $90^{\circ} \mathrm{C} / 0.1 \mathrm{Torr}$.

d. (Ttfac)Cu(7-t-BuO-NBD) (4). Yellow, crystalline (Ttfac)$\mathrm{Cu}(7-t$-BuO-NBD) was prepared in $66 \%$ yield by using a procedure analogous to that of the synthesis of compound 1 .

Anal. Calcd for $\mathrm{C}_{19} \mathrm{H}_{20} \mathrm{O}_{3} \mathrm{~F}_{3} \mathrm{CuS}$ : C, 50.83; H, 4.49. Found: C, $50.62 ; \mathrm{H}, 4.47$

NMR data $\left(\mathrm{C}_{6} \mathrm{D}_{6}, 18^{\circ} \mathrm{C}\right):{ }^{1} \mathrm{H} \delta 7.26(\mathrm{~d}, 1 \mathrm{H}, J=3.2 \mathrm{~Hz}$, thienyl $H$ on Ttfac), 6.85 (d, $1 \mathrm{H}, J=4.8 \mathrm{~Hz}$, thienyl $H$ on Ttfac), $6.53(\mathrm{~m}, 1 \mathrm{H}$, thienyl $H$ on Ttfac), $6.51(\mathrm{~s}, 1 \mathrm{H}$, methine $\mathrm{CH}$ on Ttfac), 6.09 (br, s, 2H, noncoordinated $\mathrm{CH}=\mathrm{CH}$ on 7-tBuO-NBD), 4.99 (br, s, $2 \mathrm{H}$, coordinated $\mathrm{CH}=\mathrm{CH}$ on $7-t-\mathrm{BuO}$ $\mathrm{NBD}$ ), 3.39 (br, s, 1H, bridge $H$ on 7-t-BuO-NBD), 3.06 (br, s, $2 \mathrm{H}$, bridgehead $\mathrm{H}$ on $7-t-\mathrm{BuO}-\mathrm{NBD}), 0.97$ (s, $9 \mathrm{H}, \mathrm{C}\left(\mathrm{CH}_{3}\right)_{3}$ on 7-t-BuO-NBD) ppm. ${ }^{13} \mathrm{C}\left\{{ }^{1} \mathrm{H}\right\} \delta 183.0$ (s, CO on Ttfac), 172.5 $\left(\mathrm{q}, J_{\mathrm{FC}}=32 \mathrm{~Hz}, \mathrm{CF}_{3} \mathrm{CO}\right.$ on Ttfac), 146.8 (s, thienyl $\mathrm{C}$ on Ttfac), 139.6 (s, noncoordinated $C H=C H$ on $7-t-\mathrm{BuO}-\mathrm{NBD}) 132.7$ (s, thienyl $C$ on Ttfac), 130.5 (s, thienyl $C$ on Ttfac), 120.8 (q, $J_{\mathrm{FC}}$ $=284 \mathrm{~Hz}, \mathrm{CF}_{3}$ on Ttfac), 98.2 (s, coordinated $\mathrm{CH}=\mathrm{CH}$ on $7-t$ BuO-NBD), $92.3(\mathrm{~s}$, bridge $C$ on $7-t$-BuO-NBD), $91.5(\mathrm{~s}$, methine $C \mathrm{H}$ on Ttfac), 76.5 (s, $\left(\mathrm{CH}_{3}\right)_{3} \mathrm{C}$ on $7-t$-BuO-NBD), 55.9 (s, bridgehead $C$ on $7-t-\mathrm{BuO}-\mathrm{NBD}), 28.5\left(\mathrm{~s},\left(\mathrm{CH}_{3}\right)_{3} \mathrm{C}\right.$ on $7-t-$ BuO-NBD) ppm; ${ }^{19} \mathrm{~F}\left\{{ }^{1} \mathrm{H}\right\} \delta-75.3(\mathrm{~s}) \mathrm{ppm}$.

IR data ( $\mathrm{KBr}$ disk): $3752(\mathrm{w}), 2976(\mathrm{w}), 1602(\mathrm{~s}), 1536(\mathrm{~s})$, $1516(\mathrm{~m}), 1464(\mathrm{~m}), 1412(\mathrm{~m}), 1354(\mathrm{~m}), 1304(\mathrm{~s}), 1232(\mathrm{~m})$, $1172(\mathrm{~s}), 1144(\mathrm{~s}), 1080(\mathrm{~s}), 1036(\mathrm{w}), 930(\mathrm{w}), 888(\mathrm{w}), 782$ (m), $728(\mathrm{w}), 688(\mathrm{~m}), 668(\mathrm{w}), 584(\mathrm{w}) \mathrm{cm}^{-1}$.

Mass spectral data $\left(\mathrm{FAB}^{+}, \mathrm{m} / \mathrm{z}\right): 568,12.2\left[\mathrm{Cu}_{2}\left(\mathrm{Ttfac}_{2}\right]^{++}\right.$; $448,34.1[\mathrm{Cu}(\mathrm{Ttfac})(7-t-\mathrm{BuO}-\mathrm{NBD})]^{0+} ; 392,85.2[\mathrm{Cu}(\mathrm{Ttfac})(7-$ HO-NBD) $]^{++} ; 285,24.5$ [HCu(Ttfac) ${ }^{0+} ; 227,67.3[\mathrm{Cu}(7-t-\mathrm{BuO}-$ NBD) $]^{++} ; 171,39.2[\mathrm{Cu}(7-\mathrm{HO}-\mathrm{NBD})]^{++} ; 141,18.0\left[\mathrm{Cu}\left(\mathrm{C}_{6} \mathrm{H}_{6}\right)\right]^{++}$; $111,21.8\left[\mathrm{C}_{5} \mathrm{H}_{3} \mathrm{OS}\right]^{\circ+} ; 107,18.8[(7-\mathrm{O}-\mathrm{NBD})]^{++} ; 92,26.2[\mathrm{NBD}]^{+*}$; $91,100\left[\mathrm{C}_{7} \mathrm{H}_{7}\right]^{++} ; 63,7.0[\mathrm{Cu}]^{++} ; 57,24.8\left[\mathrm{C}_{4} \mathrm{H}_{9}\right]^{++}$.

Mp: $115^{\circ} \mathrm{C} \mathrm{dec}$, sublimation temperature: $90^{\circ} \mathrm{C} / 0.1$ Torr.

e. (Btfac)Cu(7-t-BuO-NBD) (5). Yellow, crystalline (Btfac) $\mathrm{Cu}(7-t-\mathrm{BuO}-\mathrm{NBD})$ was prepared in $63 \%$ yield by using a procedure analogous to that of synthesis of compound 1 .

Anal. Calcd for $\mathrm{C}_{21} \mathrm{H}_{22} \mathrm{O}_{3} \mathrm{~F}_{3} \mathrm{Cu}: \mathrm{C}, 56.94 ; \mathrm{H}, 5.01$. Found: C, 56.94; H, 5.02 .

NMR data $\left(\mathrm{C}_{6} \mathrm{D}_{6}, 18^{\circ} \mathrm{C}\right):{ }^{1} \mathrm{H} \delta 7.86(\mathrm{~m}, 2 \mathrm{H}$, phenyl $H$ on Btfac), $7.07(\mathrm{~m}, 3 \mathrm{H}$, phenyl $H$ on Btfac), $6.59(\mathrm{~s}, 1 \mathrm{H}$, methine $\mathrm{CH}$ on Btfac), 6.16 (br, s, $2 \mathrm{H}$, noncoordinated $\mathrm{CH}=\mathrm{CH}$ on 7-t. $\mathrm{BuO}-\mathrm{NBD}$ ), $5.03(\mathrm{br}, \mathrm{s}, 2 \mathrm{H}$, coordinated $\mathrm{CH}=\mathrm{CH}$ on $7-t-\mathrm{BuO}$ -

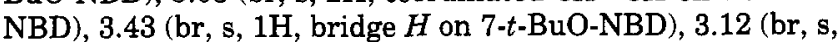
$2 \mathrm{H}$, bridgehead $\mathrm{H}$ on $7-t-\mathrm{BuO}-\mathrm{NBD}), 0.96\left(\mathrm{~s}, 9 \mathrm{H}, \mathrm{C}\left(\mathrm{CH}_{3}\right)_{3}\right.$ on 7-t-BuO-NBD) ppm; ${ }^{13} \mathrm{C}\left\{{ }^{1} \mathrm{H}\right\} \delta 189.9$ (s, $\mathrm{C}_{6} \mathrm{H}_{5} \mathrm{CO}$ on $\mathrm{Btfac}$ ), $173.0\left(\mathrm{q}, J_{\mathrm{FC}}=32.0 \mathrm{~Hz}, \mathrm{CF}_{3} \mathrm{CO}\right.$ on $\mathrm{Btfac}$ ), 140.4 (s, phenyl $C$ on Btfac), 139.8 (s, noncoordinated $\mathrm{CH}=\mathrm{CH}$ on 7-t-BuO-NBD), 132.6 (s, phenyl $C$ on Btfac), $121.3\left(\mathrm{q}, J_{\mathrm{FC}}=284.3 \mathrm{~Hz}, C \mathrm{~F}_{3}\right.$ on Btfac), 97.9 (s, coordinated $\mathrm{CH}=\mathrm{CH}$ on $7-t$-BuO-NBD), 92.3 (s, methine $\mathrm{CH}$ on $\mathrm{Btfac}$ ), 92.0 (s, bridge $C$ on $7-t$-BuO-NBD), $76.6\left(\mathrm{~s},\left(\mathrm{CH}_{3}\right)_{3} C\right.$ on $\left.7-t-\mathrm{BuO}-\mathrm{NBD}\right), 56.0$ (s, bridgehead $C$ on 7-t-BuO-NBD), $28.5\left(\mathrm{~s},\left(\mathrm{CH}_{3}\right)_{3} \mathrm{C}\right.$ on 7 -t-BuO-NBD) ppm; ${ }^{19} \mathrm{~F}$. $\left\{{ }^{1} \mathrm{H}\right\} \delta-75.2(\mathrm{~s}) \mathrm{ppm}$.

IR data (KBr disk): $3752(\mathrm{w}), 2982(\mathrm{w}), 1612(\mathrm{~s}), 1587(\mathrm{~m})$, $1540(\mathrm{~m}), 1482(\mathrm{~m}), 1377(\mathrm{w}), 1292(\mathrm{~s}), 1257(\mathrm{~m}), 1178(\mathrm{~s}), 1152$ (s), $1088(\mathrm{~m}), 898$ (w), $764(\mathrm{w}), 686(\mathrm{w}), 658(\mathrm{w}), 596(\mathrm{w}) \mathrm{cm}^{-1}$.

Mass spectral data $\left(\mathrm{FAB}^{+}, \mathrm{m} / z\right): 720,12.4\left[\mathrm{Cu}_{2}(\mathrm{Btfac})_{2}(7-\right.$ $t$-BuO-NBD) $]^{++} ; 442,59.6\left[\mathrm{Cu}(\mathrm{Btfac})(7-t \text {-BuO-NBD) }]^{++} ; 386,100\right.$ $[\mathrm{Cu}(\mathrm{Btfac})(7-\mathrm{HO}-\mathrm{NBD})]^{\circ+} ; 279,51.4[\mathrm{HCu}(\mathrm{Btfac})]^{\circ+} ; 227,87.2$ $[\mathrm{Cu}(7-t-\mathrm{BuO}-\mathrm{NBD})]^{++} ; 216,12.5\left[\mathrm{HBtfac}^{++} ; 171,41.1[\mathrm{Cu}(7-\right.$
HO-NBD) $]^{++} ; 141,15.2\left[\mathrm{Cu}\left(\mathrm{C}_{6} \mathrm{H}_{6}\right)\right]^{++} ; 107,15.5[(7-\mathrm{O}-\mathrm{NBD})]^{+*}$; $92,12.9[\mathrm{NBD}]^{++} ; 91,83.3\left[\mathrm{C}_{7} \mathrm{H}_{7}\right]^{++} ; 77,9.5\left[\mathrm{C}_{6} \mathrm{H}_{5}\right]^{\circ+} ; 63,4.6$ $[\mathrm{Cu}]^{++} ; 57,17.6\left[\mathrm{C}_{4} \mathrm{H}_{9}\right]^{++}$.

Mp: $116^{\circ} \mathrm{C}$, sublimation temperature: $90^{\circ} \mathrm{C} / 0.12$ Torr.

f. (fod)Cu(7-t-BuO-NBD) (6). Yellow, crystalline (fod)$\mathrm{Cu}(7-t-\mathrm{BuO}-\mathrm{NBD})$ was prepared in $53 \%$ with a procedure analogous to that for compound 1 .

Anal. Caled for $\mathrm{C}_{21} \mathrm{H}_{26} \mathrm{O}_{3} \mathrm{~F}_{7} \mathrm{Cu}: \mathrm{C}, 48.23 ; \mathrm{H}, 5.01$. Found: C, 48.10; H, 5.04.

NMR data $\left(\mathrm{C}_{6} \mathrm{D}_{6}, 18{ }^{\circ} \mathrm{C}\right):{ }^{1} \mathrm{H} \delta 6.15$ (s, $1 \mathrm{H}, \mathrm{CH}$ on fod), 6.10 (br, s, $2 \mathrm{H}$, noncoordinated $\mathrm{CH}=\mathrm{CH}$ on $7-t-\mathrm{BuO}-\mathrm{NBD}), 4.93(\mathrm{br}$, $\mathrm{s}, 2 \mathrm{H}$, coordinated $\mathrm{CH}=\mathrm{CH}$ on $7-t-\mathrm{BuO}-\mathrm{NBD}$ ), 3.39 (br, $\mathrm{s}, 1 \mathrm{H}$, bridge $H$ on 7-t-BuO-NBD), $3.03(\mathrm{br}, \mathrm{s}, 2 \mathrm{H}$, bridgehead $H$ on 7-t-BuO-NBD), 1.10 (s, 9H, C(CH $)_{3}$ on fod), 0.97 (s, $9 \mathrm{H}$, $\mathrm{C}\left(\mathrm{CH}_{3}\right)_{3}$ on $7-t$-BuO-NBD) ppm; ${ }^{13} \mathrm{C}\left\{{ }^{1} \mathrm{H}\right\} \delta 206.1$ (s, $\left(\mathrm{CH}_{3}\right)_{3} \mathrm{CCO}$ on fod), $172.9\left(\mathrm{t}, J_{\mathrm{FC}}=22.6 \mathrm{~Hz}, \mathrm{CF}_{2} \mathrm{CO}\right.$ on fod), $139.8(\mathrm{~s}$, noncoordinated $C H=C H$ on $7-t-B u O-N B D), 123.0-104.9(\mathrm{~m}$, $\mathrm{CF}_{2} \mathrm{CF}_{2} \mathrm{CF}_{3}$ on fod), 97.5 (s, coordinated $\mathrm{CH}=\mathrm{CH}$ on $7-t-\mathrm{BuO}$ NBD), 92.4 (s, $C H$ on fod), 92.1 (s, bridge $C$ on $7-t-\mathrm{BuO}-\mathrm{NBD}$ ), $76.4\left(\mathrm{~s},\left(\mathrm{CH}_{3}\right)_{3} \mathrm{C}\right.$ on $\left.7-t-\mathrm{BuO}-\mathrm{NBD}\right), 55.9(\mathrm{~s}$, bridgehead $\mathrm{C}$ on 7-t-BuO-NBD), 43.0 (s, $C\left(\mathrm{CH}_{3}\right)_{3}$ on fod), $28.6\left(\mathrm{~s}, \mathrm{C}\left(\mathrm{CH}_{3}\right)_{3}\right.$ on fod), 28.5 (s, $\mathrm{C}\left(\mathrm{CH}_{3}\right)_{3}$ on $\left.7-t-\mathrm{BuO}-\mathrm{NBD}\right) \mathrm{ppm} ;{ }^{19} \mathrm{~F}\left\{{ }^{1} \mathrm{H}\right\} \delta-80.5$ (s), $-119.0(\mathrm{~s}),-126.2(\mathrm{~s}) \mathrm{ppm}$.

IR data (KBr disk): $2986(\mathrm{~m}), 1627(\mathrm{~s}), 1581(\mathrm{~m}), 1511(\mathrm{~m})$, $1480(\mathrm{~s}), 1401(\mathrm{w}), 1355(\mathrm{~m}), 1231$ (s), $1198(\mathrm{~s}), 1125(\mathrm{~m}), 1083$ (s), 1028 (w), $918(w), 793(w), 697(\mathrm{~m}), \mathrm{cm}^{-1}$.

Mass spectral data $\left(\mathrm{EI}^{+}, \mathrm{m} / z\right): 522,2.3[\mathrm{Cu}($ fod $)(7-t$-BuONBD $)]^{++} ; 466,3.1[\mathrm{Cu}(\text { fod })(7-\mathrm{HO}-\mathrm{NBD})]^{\circ+} ; 359,4.9[\mathrm{HCu}(\text { fod })]^{0+}$; $301,15.7\left[\mathrm{Cu}\left(\mathrm{C}_{3} \mathrm{~F}_{7} \mathrm{C}(\mathrm{O}) \mathrm{CH}-\mathrm{C}(\mathrm{O})\right)\right]^{++} ; 239,13.7\left[\mathrm{C}_{3} \mathrm{~F}_{7} \mathrm{C}(\mathrm{O}) \mathrm{CHC}-\right.$ (O) $]^{++} ; 227,10.0[\mathrm{Cu}(7-t-\mathrm{BuO}-\mathrm{NBD})]^{\circ+} ; 171,7.4[\mathrm{Cu}(7-\mathrm{HO}-$ $\mathrm{NBD})]^{++} ; 149,7.6\left[\left(7-\left(\mathrm{CH}_{3}\right)_{2} \mathrm{CO}-\mathrm{NBD}\right)\right]^{++} ; 141,5.4\left[\mathrm{Cu}\left(\mathrm{C}_{6} \mathrm{H}_{6}\right)\right]^{0+}$; 108, 37.1 [(7-HO-NBD) $]^{\circ+} ; 107,27.6[(7-\mathrm{O}-\mathrm{NBD})]^{++} ; 92,11.6$ $\left[\mathrm{NBD}^{++} ; 91,97.0\left[\mathrm{C}_{7} \mathrm{H}_{7}\right]^{++} ; 79,63.8\left[\mathrm{C}_{6} \mathrm{H}_{7}\right]^{++} ; 78,21.0\left[\mathrm{C}_{6} \mathrm{H}_{6}\right]^{++}\right.$; $77,27.1\left[\mathrm{C}_{6} \mathrm{H}_{5}\right]^{++} ; 69,24.4\left[\mathrm{CF}_{3}\right]^{++} 63,11.3[\mathrm{Cu}]^{++} ; 57,100$ $\left[\mathrm{C}_{4} \mathrm{H}_{9}\right]^{++}$

$\mathrm{Mp}: 115^{\circ} \mathrm{C}$ dec, sublimation temperature: $85^{\circ} \mathrm{C} / 0.1 \mathrm{Torr}$.

X-ray Single-Crystal Structural Determination. Three examples of the title compounds were characterized structurally in the solid state by single-crystal X-ray diffraction. All diffraction data were collected on an Enraf-Nonius CAD-4 diffractometer with monochromated Mo $\mathrm{K} \alpha$ radiation $(\lambda=$ $0.70930 \AA$ ) using $\Theta / 2 \Theta$ scan mode. Crystal, collection, and refinement data are summarized in Table 1.

a. (hfac)Cu(7-t-BuO-NBD) (1). Bright yellow crystals of (hfac) $\mathrm{Cu}(7-t-\mathrm{BuO}-\mathrm{NBD})$ were grown by crystallization from hexane solution at $-20^{\circ} \mathrm{C}$, and a single crystal of dimensions $0.40 \times 0.50 \times 0.50 \mathrm{~mm}^{3}$ was selected for X-ray analysis. Cell parameters were determined from a fit of 25 reflections $(23.40$ $<2 \Theta<27.60^{\circ}$ ). All data were corrected for Lorentz and polarization effects and for effects of absorption. A total of 3228 reflections was collected, but only 2586 unique reflections with $I>2 \sigma(I)$ were used for structural solution and refinement. The structure was solved by the heavy-atom method and refined by full-matrix least-squares methods based on $F$ values. All non-hydrogen atoms were refined with anisotropic thermal parameters. The atomic scattering factors were taken from International Tables for X-ray Crystallography. ${ }^{15}$ The final agreement factors are $R_{\mathrm{F}}=0.042, R_{\mathrm{w}}=0.042$ with $w=$ $1 / \sigma^{2}(F)$.

b. (Ttfac)Cu(7-t-BuO-NBD) (4). Light yellow crystals of (Ttfac)Cu(7-t-BuO-NBD) were grown by crystallization from hexane solution at $-20^{\circ} \mathrm{C}$ and a single crystal of dimensions $0.50 \times 0.50 \times 0.70 \mathrm{~mm}^{3}$ was selected for X-ray analysis. Cell parameters were determined from a fit of 25 reflections (19.42 $<2 \Theta<25.38^{\circ}$ ). All data were corrected for Lorentz and polarization effects and for effects of absorption. A total of 3495 reflections was collected, but only 2481 unique reflections with $I>2 o(I)$ were used for structural solution and refinement. The structure was solved by the heavy-atom method and

(15) International Tables for $X$-ray Crystallography; Kynoch Press: Birmingham, U.K., 1974; Vol. IV. 
Table 1. Summary of Crystallographic Data for Compounds 1, 4, and 5

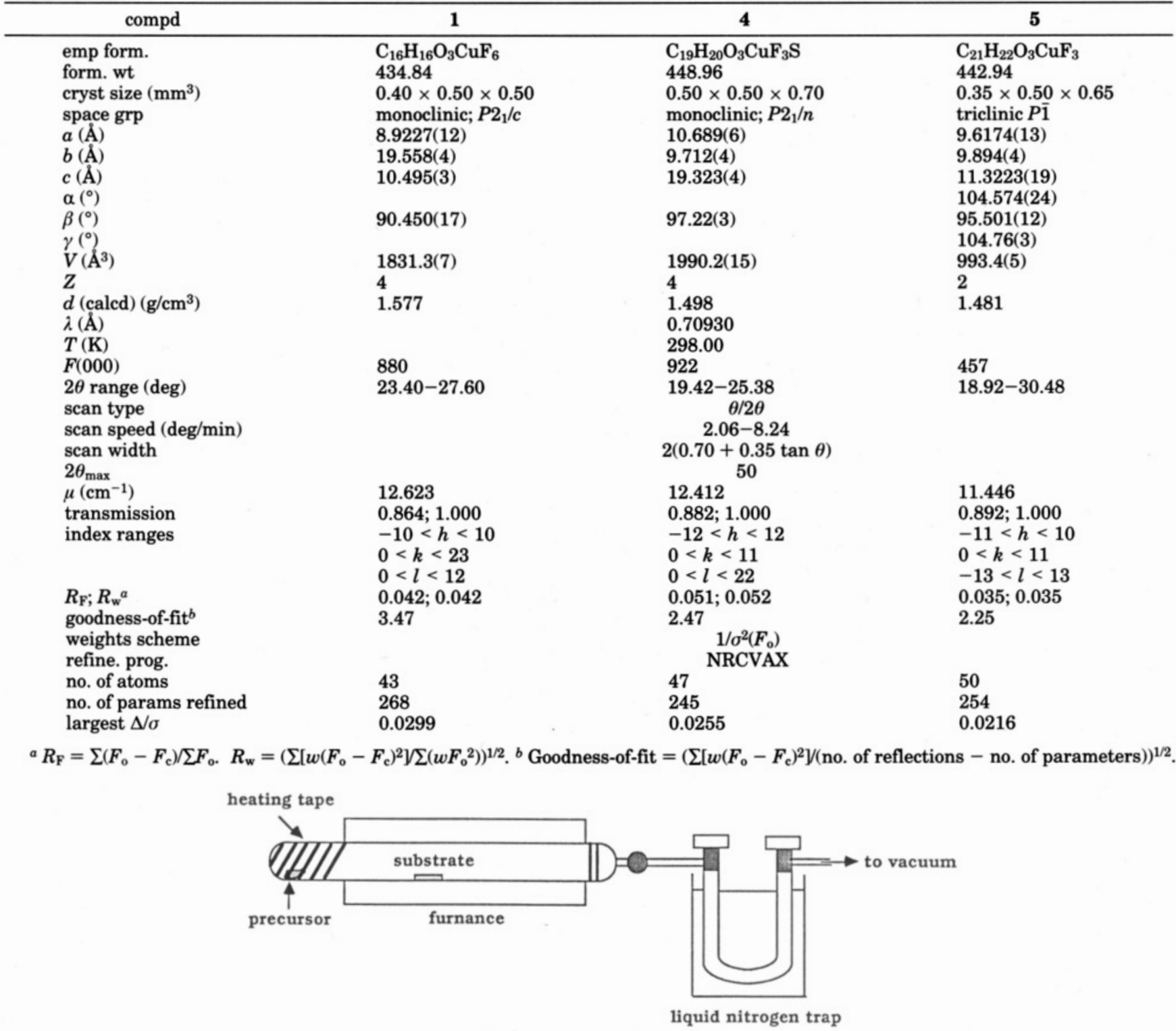

Figure 1. Schematic plot of hot-wall CVD reactor.

refined by full-matrix least-squares methods based on $F$ values. All non-hydrogen atoms were refined with anisotropic thermal parameters. The atomic scattering factors were taken from International Tables for X-ray Crystallography. ${ }^{15}$ The final agreement factors are $R_{\mathrm{F}}=0.051, R_{\mathrm{w}}=0.052$ with $w=$ $1 / \sigma^{2}(F)$.

c. (Btfac)Cu(7-t-Bu0-NBD) (5). Yellow crystals of (Btfac) $\mathrm{Cu}(7-t$-BuO-NBD) were grown by crystallization from hexane solution at $-20^{\circ} \mathrm{C}$, and a single crystal of dimensions $0.35 \times 0.50 \times 0.65 \mathrm{~mm}^{3}$ was selected for X-ray analysis. Cell parameters were determined from a fit of 25 reflections (18.92 $<2 \Theta<30.48^{\circ}$ ). All data were corrected for Lorentz and polarization effects and for effects of absorption. A total of 3499 reflections was collected, but only 2940 unique reflections with $I>2 \sigma(I)$ were used for structural solution and refinement. The structure was solved by the heavy-atom method and refined by full-matrix least-squares based on $F$ values. All non-hydrogen atoms were refined by full-matrix least-squares based on $F$ values. All non-hydrogen atoms were refined with anisotropic thermal parameters. The atomic scattering factors were taken from International Tables for X-ray Crystallography. ${ }^{15}$ The final agreement factors are $R_{\mathrm{F}}=0.035, R_{\mathrm{w}}=$ 0.035 with $w=1 / \sigma^{2}(F)$.

Chemical Vapor Deposition of Copper Films from Compound 1. CVD experiments were conducted in the hot- wall reactor shown in Figure 1. Substrates of two types, $\mathrm{Si}$ (100) wafers coated with $1000 \AA$ of $\mathrm{Pt}$ and with $2000 \AA$ of thermally grown $\mathrm{SiO}_{2}$, were used. The substrates, typically $1.5 \mathrm{~cm} \times 1.5 \mathrm{~cm}^{2}$, were cleaned with deionized water, acetone, and 1,1,1-trichloroethane and then heated to $100^{\circ} \mathrm{C}$ for $20 \mathrm{~min}$ before use. The CVD reactor was charged with (hfac)Cu(7-t$\mathrm{BuO}-\mathrm{NBD}$ ) (approximately $0.5 \mathrm{~g}$ ) and a substrate in a glovebox with nitrogen atmosphere for each experiment. The assembled reactor was attached to a U-trap that was cooled with liquid $\mathrm{N}_{2}$ and connected to a vacuum line. While the system was evacuated ( 0.1 Torr), the deposition zone was preheated with a furnance for $1 \mathrm{~h}$ to attain the deposition temperature and the precursor remained at room temperature. The precursor was then warmed to $80^{\circ} \mathrm{C}$ with a heating tape to transfer into the deposition zone and deposition was continued until all precursor had evaporated. Deposition was undertaken over a temperature range $140-260{ }^{\circ} \mathrm{C}$ at $30^{\circ} \mathrm{C}$ intervals. No carrier gas was used in these experiments. Film resistivities were measured with a four-point probe.

\section{Results and Discussion}

1. Synthesis of ( $\beta$-Diketonate)Cu(7-t-BuO-NBD) Complexes. The title compounds were prepared from reactions of $\mathrm{CuCl}$ with the corresponding $\beta$-diketonate 


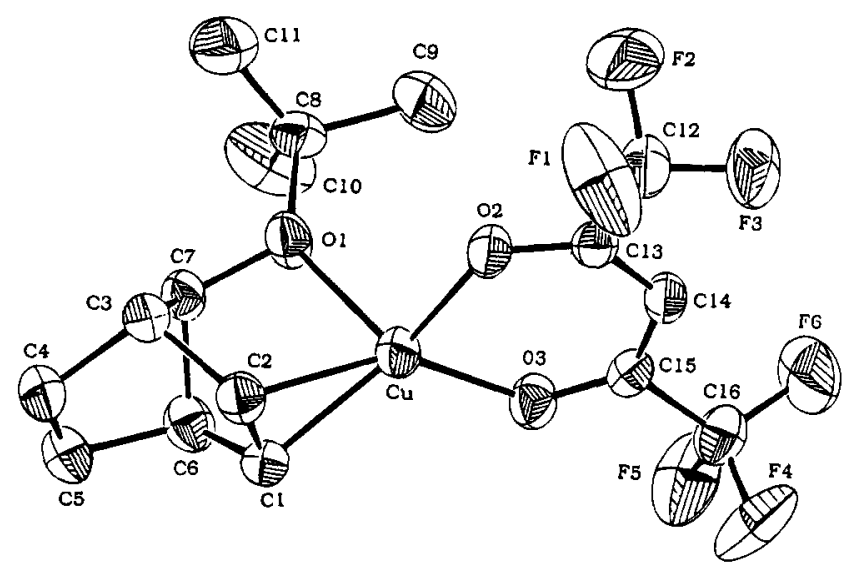

Figure 2. ORTEP drawing of (hfac) $\mathrm{Cu}(7-t-\mathrm{BuO}-\mathrm{NBD})$ (1).

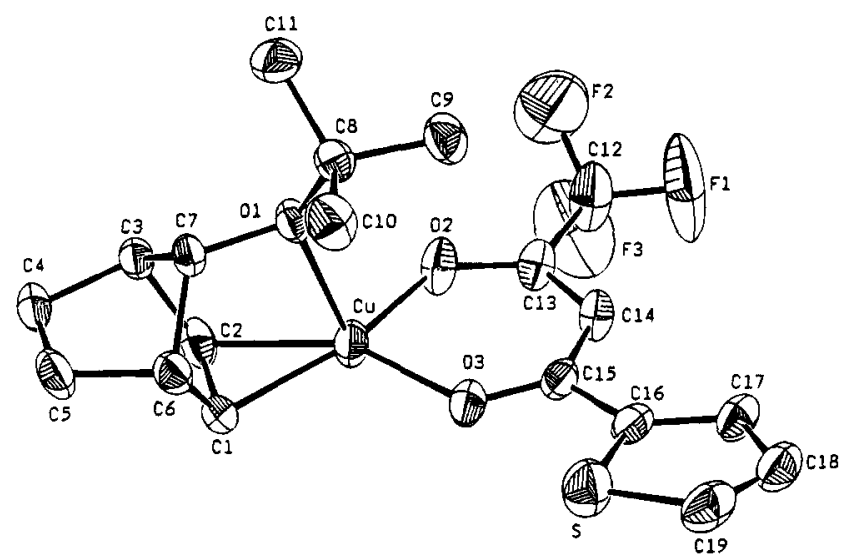

Figure 3. ORTEP drawing of (Ttfac) Cu(7-t-BuO-NBD) (4).

salts of sodium in the presence of 7-t-BuO-NBD according to eq 2 . The reactions were carried out in ethereal

$$
\begin{aligned}
\mathrm{CuCl}+ & \mathrm{Na}(\beta \text {-diketonate })+7-t-\mathrm{BuO}-\mathrm{NBD} \rightarrow \\
& (\beta \text {-diketonate }) \mathrm{Cu}(7-t-\mathrm{BuO}-\mathrm{NBD})+\mathrm{NaCl}
\end{aligned}
$$

solutions at room temperature, and products were isolated on extraction with hexane and removal of volatile species in vacuo. A similar method was used to prepare analogous complexes of 1,5-cyclooctadiene $(1,5-C O D) .{ }^{11 b}$ These compounds are yellow to white solids that can be sublimed at temperatures above 75 ${ }^{\circ} \mathrm{C}$ under a reduced pressure (about 0.1 Torr). They are thermally robust and moderately sensitive to air and moisture. All compounds gave satisfactory elemental analyses and were characterized by solution ${ }^{1} \mathrm{H},{ }^{13} \mathrm{C}$, and ${ }^{19} \mathrm{~F}$ NMR, IR, and mass spectroscopic methods.

2. Single-Crystal Structural Determination of Compounds 1, 4, and 5. The molecular structures of (hfac)Cu(7-t-BuO-NBD) (1), (Ttfac)Cu(7-t-BuO-NBD) (4), and (Btfac)Cu(7-t-BuO-NBD) (5) were determined in the solid state by single-crystal X-ray diffraction analyses. ORTEP diagrams showing the atomic labeling scheme of these molecules are presented in Figures $2-4$. The crystal, collection, and refinement data are summarized in Table 1; selected bond lengths and bond angles are given in Tables 2 and 3 . Unlike the dinuclear norbornadiene derivative, $[(\mathrm{hfac}) \mathrm{Cu}]_{2}$ (norbornadiene), ${ }^{11 \mathrm{a}}$ these compounds are mononuclear and the central copper atom is coordinated by the chelating $\beta$-diketonate ligand through two oxygen atoms and the 7-t-BuO-NBD through one $\mathrm{C}=\mathrm{C}$ double bond and an oxygen atom. The same coordination mode for the 7-t-BuO-NBD ligand is

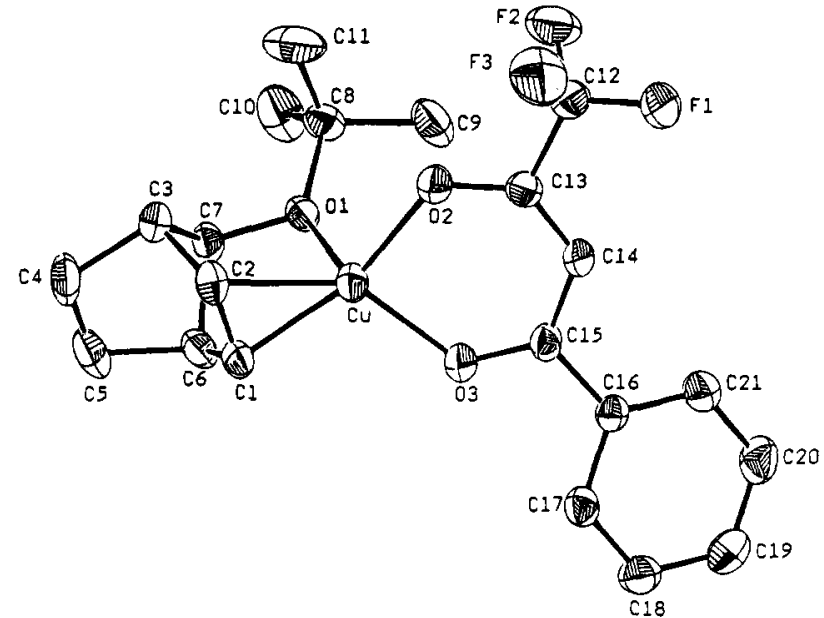

Figure 4. ORTEP drawing of (Btfac)Cu(7-t-BuO-NBD) (5).

Table 2. Selected Bond Lengths $(\AA)$ for Compounds 1, 4, and 5

\begin{tabular}{cccc}
\hline compd & $\mathbf{1}$ & $\mathbf{4}$ & $\mathbf{5}$ \\
\hline $\mathrm{Cu}-\mathrm{O}(1)$ & $2.249(2)$ & $2.344(3)$ & $2.365(2)$ \\
$\mathrm{Cu}-\mathrm{O}(2)$ & $1.976(2)$ & $1.943(3)$ & $1.953(2)$ \\
$\mathrm{Cu}-\mathrm{O}(3)$ & $1.988(2)$ & $1.962(3)$ & $1.966(2)$ \\
$\mathrm{Cu}-\mathrm{C}(1)$ & $2.011(3)$ & $1.991(5)$ & $1.987(3)$ \\
$\mathrm{Cu}-\mathrm{C}(2)$ & $2.029(3)$ & $2.014(4)$ & $2.033(3)$ \\
$\mathrm{C}(1)-\mathrm{C}(2)$ & $1.346(5)$ & $1.359(8)$ & $1.354(4)$ \\
$\mathrm{C}(4)-\mathrm{C}(5)$ & $1.294(6)$ & $1.299(8)$ & $1.311(6)$
\end{tabular}

Table 3. Selected Bond Angles (deg) for Compounds 1, 4, and 5

\begin{tabular}{crrr}
\hline compd & \multicolumn{1}{c}{$\mathbf{1}$} & \multicolumn{1}{c}{$\mathbf{4}$} & \multicolumn{1}{c}{$\mathbf{5}$} \\
\hline $\mathrm{O}(1)-\mathrm{Cu}-\mathrm{O}(2)$ & $105.4(1)$ & $104.2(1)$ & $101.8(1)$ \\
$\mathrm{O}(1)-\mathrm{Cu}-\mathrm{O}(3)$ & $109.2(1)$ & $99.7(1)$ & $104.4(1)$ \\
$\mathrm{O}(1)-\mathrm{Cu}-\mathrm{C}(1)$ & $81.6(1)$ & $81.8(2)$ & $80.5(1)$ \\
$\mathrm{O}(1)-\mathrm{Cu}-\mathrm{C}(2)$ & $81.2(1)$ & $79.8(2)$ & $81.3(1)$ \\
$\mathrm{O}(2)-\mathrm{Cu}-\mathrm{O}(3)$ & $93.3(1)$ & $95.2(1)$ & $94.0(1)$ \\
$\mathrm{O}(2)-\mathrm{Cu}-\mathrm{C}(1)$ & $152.7(1)$ & $153.6(2)$ & $159.3(1)$ \\
$\mathrm{O}(2)-\mathrm{Cu}-\mathrm{C}(2)$ & $115.0(1)$ & $115.2(2)$ & $120.2(1)$ \\
$\mathrm{O}(3)-\mathrm{Cu}-\mathrm{C}(1)$ & $109.5(1)$ & $109.4(2)$ & $105.4(1)$ \\
$\mathrm{O}(3)-\mathrm{Cu}-\mathrm{C}(2)$ & $146.6(1)$ & $149.0(2)$ & $143.8(1)$ \\
$\mathrm{C}(1)-\mathrm{Cu}-\mathrm{C}(2)$ & $38.9(2)$ & $39.7(2)$ & $39.3(1)$ \\
$\mathrm{Cu}-\mathrm{O}(1)-\mathrm{C}(7)$ & $99.6(2)$ & $97.1(2)$ & $96.3(1)$ \\
$\mathrm{Cu}-\mathrm{C}(1)-\mathrm{C}(2)$ & $71.3(2)$ & $71.1(3)$ & $72.2(2)$ \\
$\mathrm{Cu}-\mathrm{C}(2)-\mathrm{C}(1)$ & $69.8(2)$ & $69.3(3)$ & $68.5(2)$
\end{tabular}

reported in the structure of $(7-t-\mathrm{BuO}-\mathrm{NBD})_{2} \mathrm{Mo}(\mathrm{CO})_{2} \cdot{ }^{16}$ The bond distances and angles within the $\beta$-diketonate ligand are similar to those of other $\beta$-diketonate copper(I) complexes according to previous reports. ${ }^{10-12}$ The coordination geometry about copper is best described as a highly distorted tetrahedron that resembles that of (hfac)Cu(1,5-COD). ${ }^{11 \mathrm{~b}}$ The atoms $\mathrm{O}(2), \mathrm{O}(3), \mathrm{C}(1)$, and $\mathrm{C}(2)$ are mutually coplanar, and the copper atom lies slightly (for example, $0.091 \AA$ for compound 1) above the plane. The $O(1)$ atom occupies the fourth coordination site with a relatively weak interaction to copper atom $(\mathrm{Cu}-\mathrm{O}(1)$ distance $=2.249(2), 2.344(3)$, and $2.365(2) \AA$ for compounds 1,4 , and 5, respectively) compared with bonding between copper and oxygens of $\beta$-diketonate ligands in the same molecule $(\mathrm{Cu}-\mathrm{O}(2)$, $\mathrm{Cu}-\mathrm{O}(3)$ bond distances are 1.94-1.99 $\AA$ for compounds $\mathbf{1}, \mathbf{4}$, and 5 ), and with $\mathrm{Cu}-\mathrm{O}$ bonding in the molecule $(t-\mathrm{BuOCu})_{4}$ (average bond length is $\left.1.85 \AA\right){ }^{17}$ Thus, the structures are best described as " $3+1$ " coordination of the copper center also observed in the molecular struc-

(16) Chow, T. J.; Chao, Y,-S.; Liu, L.-K. J. Am. Chem. Soc. 1987, 109,797 .

(17) Greiser, T.; Weiss, E. Chem. Ber. 1976, 109, 3142. 
Table 4. Chemical Shifts ${ }^{a}$ of Olefinic ${ }^{1} \mathrm{H}$ and ${ }^{13} \mathrm{C}$ NMR Resonances for 7-t-BuO-NBD and Compounds 1-6

\begin{tabular}{|c|c|c|c|c|}
\hline \multirow{2}{*}{ compd } & \multicolumn{4}{|c|}{$\delta\left(\Delta \delta^{b}\right)(\mathrm{ppm})$} \\
\hline & \multicolumn{2}{|c|}{${ }^{1} \mathrm{H}$} & \multicolumn{2}{|c|}{${ }^{13} \mathrm{C}$} \\
\hline $\begin{array}{c}\text { 7-t-BuO- } \\
\text { NBD }\end{array}$ & 6.54 & 6.56 & 137.9 & 140.6 \\
\hline 1 & $5.02(-1.52)$ & $6.04(-0.52)$ & $101.9(-36.0)$ & $139.6(-1.0)$ \\
\hline $\mathbf{2}$ & $4.96(-1.58)$ & $6.09(-0.47)$ & $97.4(-40.5)$ & $139.8(-0.8)$ \\
\hline 3 & $4.88(-1.66)$ & $6.14(-0.42)$ & $93.3(-44.6)$ & $139.9(-0.7)$ \\
\hline 4 & $4.99(-1.55)$ & $6.09(-0.47)$ & $98.2(-39.7)$ & $139.6(-1.0)$ \\
\hline $\mathbf{5}$ & $5.03(-1.51)$ & $6.16(-0.40)$ & $97.9(-40.0)$ & $139.8(-0.8)$ \\
\hline 6 & $4.93(-1.61)$ & $6.10(-0.46)$ & $97.5(-40.4)$ & $139.8(-0.8)$ \\
\hline
\end{tabular}

ture of (hfac) $\mathrm{Cu}(1,5-\mathrm{COD}) .{ }^{11 \mathrm{~b}}$ Because hfac ligand possesses two electron-withdrawing $\mathrm{CF}_{3}$ substituents, bond distances between copper and oxygen atoms of $\beta$-diketonate in compound 1 are longer than those in compounds 4 and 5. To compensate for the electron deficiency on the copper center, donation of more electron density from $\mathrm{O}(1)$ to $\mathrm{Cu}$, which resulted in a shorter (approximately $0.1 \AA$ ) $\mathrm{Cu}-\mathrm{O}(1)$ bond in compound 1 than in compounds 4 and 5 , was observed. The longer bonds of the coordinated olefins $(\mathrm{C}(1)-\mathrm{C}(2)=$ $1.346(5), 1.359(8)$, and 1.354(4) $\AA$ for compounds 1,4 , and 5 , respectively) than those of the noncoordinated olefins $(\mathrm{C}(4)-\mathrm{C}(5)=1.294(6), 1.299(8)$, and 1.311(6) $\AA$ for compounds 1,4 , and 5 , respectively) indicate the existence of $\pi$-backbonding between alkene and copper centers in these compounds (see below). No other distances and angles within the 7-t-BuO-NBD are exceptional.

3. NMR Spectroscopic Studies of ( $\beta$-Diketonate)Cu(7-t-BuO-NBD) Complexes. The integrated ${ }^{1} \mathrm{H}$ and ${ }^{13} \mathrm{C}$ NMR spectra of the title compounds are consistent with the empirical formula ( $\beta$-diketonate)$\mathrm{Cu}(7-t-\mathrm{BuO}-\mathrm{NBD})$ and exhibit olefinic resonances of the two types presented in Table 4. Both resonances have smaller chemical shifts than those of free 7-t-BuO-NBD. The signal at higher field is associated with the coor- dinated olefinic ${ }^{1} \mathrm{H}$ or ${ }^{13} \mathrm{C}$ and the other is attributed to the noncoordinated olefin. Comparisons of ${ }^{1} \mathrm{H}$ and ${ }^{13} \mathrm{C}$ chemical shifts for the free alkene and the alkene coordinating to the $\mathrm{Cu}(\mathrm{I})$ center were used to evaluate the relative contributions of $\pi$ and $\sigma$ bonding in the complexes. ${ }^{18,19}$ Variations of chemical shifts $(\Delta \delta)$ of olefinic protons and carbons for compounds 1-6 are listed in Table 4 and are much larger than those for other ( $\beta$-diketonate)(alkene) $\mathrm{Cu}(\mathrm{I})$ compounds that were found to be between +0.02 and $-0.74 \mathrm{ppm}$ and between -8.14 and $-29.04 \mathrm{ppm}$ for ${ }^{1} \mathrm{H}$ and ${ }^{13} \mathrm{C}$ signals, respectively. ${ }^{18}$ This feature implies the existence of relatively stronger $\pi$-backbonding between alkene and copper center in the title compounds. Shieldings of both coordinated olefinic ${ }^{1} \mathrm{H}$ and ${ }^{13} \mathrm{C}$ nuclei of these compounds follow the increasing order of $3>2>1$ in agreement with electron donor capabilities of $\beta$-diketonate ligands.

On the basis of the solid-state structures of compounds 4 and 5 , those containing an asymmetrically substituted $\beta$-diketonate ligand $\left(R \neq R^{\prime}\right.$, see Scheme 1$)$ are expected to exhibit bridgehead $[\mathrm{H}(3), \mathrm{H}(6)]$, coordinated olefinic $[\mathrm{H}(1), \mathrm{H}(2)]$, and noncoordinated olefinic $[\mathrm{H}(4), \mathrm{H}(5)]$ proton NMR signals of two types. This structural feature is consistent with the variabletemperature ${ }^{1} \mathrm{H}$ NMR spectra of compound 2 in $\mathrm{CD}_{2} \mathrm{Cl}_{2}$ solution are shown in Figure 5. The room-temperature NMR spectrum exhibits single bridgehead, coordinated olefinic, and noncoordinated olefinic proton signals at $\delta 3.37,5.21$, and $6.52 \mathrm{ppm}$, respectively, probably resulting from rapid dynamic rearrangement of the molecule in solution. Upon cooling, bridgehead and coordinated olefinic proton resonances decoalesced each into two signals of equal intensity. The activation barrier $\Delta G \ddagger$ of dynamic rearrangement was estimated to be $11.7 \pm 0.5 \mathrm{kcal} \cdot \mathrm{mol}^{-1}$ from the coalescence temperature (about $241 \mathrm{~K}, \Delta v=16.3 \mathrm{~Hz}$ ) of bridgehead protons. However, only one broad noncoordinated olefinic proton resonance was observed in these spectra even at a temperature of $203 \mathrm{~K}$. Similar variable-

\section{Scheme 1}
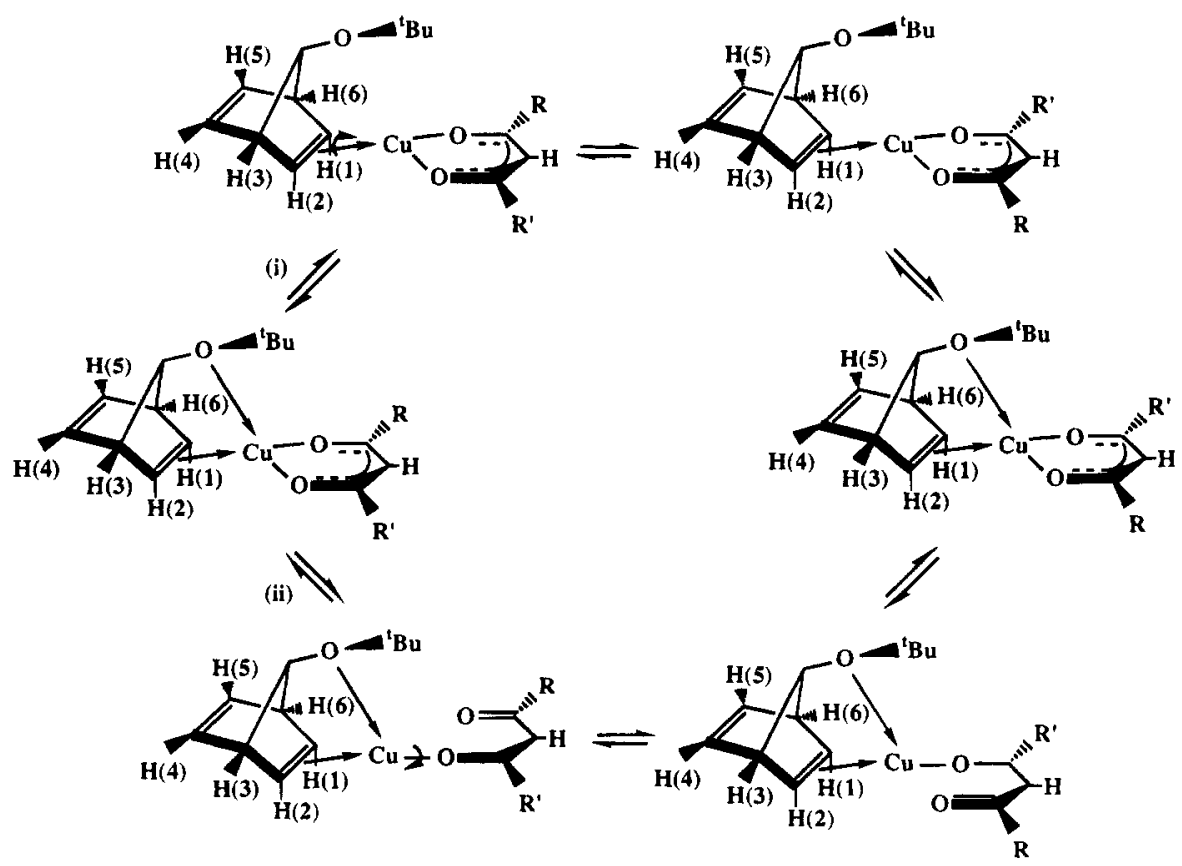


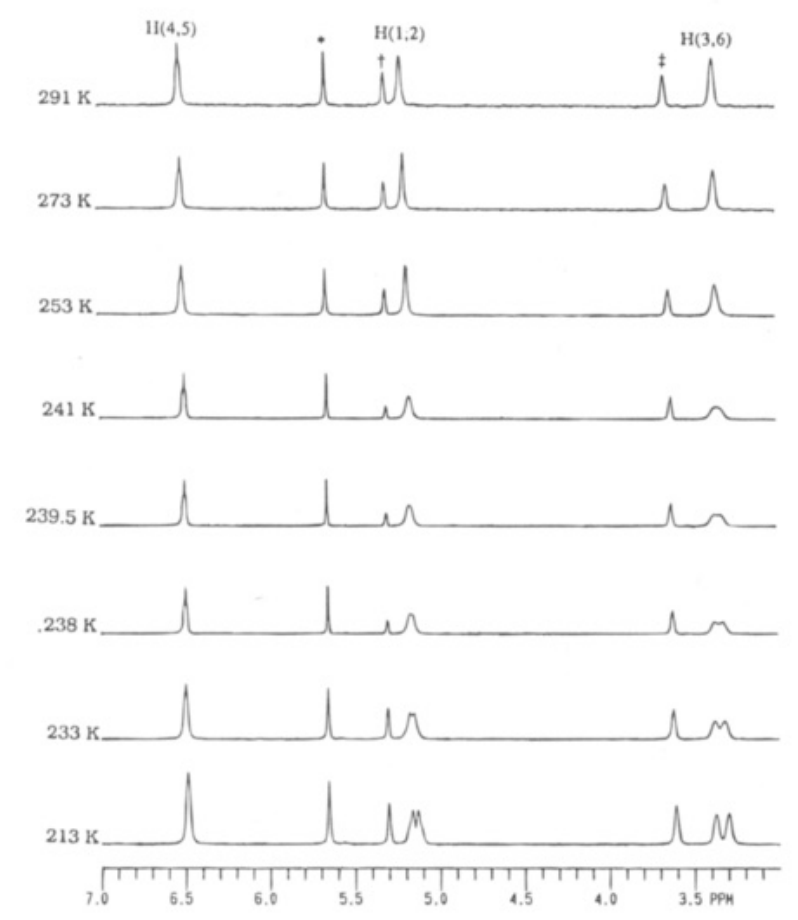

Figure 5. Variable-temperature ${ }^{1} \mathrm{H}$ NMR spectra of (tfac) $\mathrm{Cu}(7-t-\mathrm{BuO}-\mathrm{NBD})\left(*, \mathrm{CD}_{2} \mathrm{Cl}_{2} ; \dagger\right.$, methine $\mathrm{H}$ on tfac; and $\ddagger$, bridge $\mathrm{H}$ on 7 - $t$-BuO-NBD).

temperature NMR measurements (down to $203 \mathrm{~K}$ ) of compounds 4-6 were made, but the low-temperature limiting spectra were not attained. Two plausible routes to undergo the dynamic rearrangement are proposed in Scheme 1: (i) rotation of the $\mathrm{Cu}(\beta$-diketonate) moiety about the $\mathrm{Cu}$-olefin bond and (ii) rotation of the $\mathrm{Cu}(7-t-\mathrm{BuO}-\mathrm{NBD})$ moiety about the bond between $\mathrm{Cu}$ and one $\mathrm{O}$ atom of the $\beta$-diketonate ligand. Both routes involve cleavage of the $\mathrm{Cu}-\mathrm{O}$ bond. On the basis of the structures of compounds 1,4 , and 5 in the solid state, the bonds between $\mathrm{Cu}$ and $\mathrm{O}$ of $7-t-\mathrm{BuO}-\mathrm{NBD}$ are more labile than those between $\mathrm{Cu}$ and $\mathrm{O}$ of the $\beta$-diketonate in the title compounds. Exchange between the free $\beta$-diketonate anion with the coordinated $\beta$-diketonate of compound 2 has also been examined. Addition of $\mathrm{Na}$ (tfac) to a $\mathrm{CD}_{2} \mathrm{Cl}_{2}$ solution of (tfac) $\mathrm{Cu}(7-t-\mathrm{BuO}$ NBD) resulted in no exchange with coordinated tfac ligand on the ${ }^{1} \mathrm{H}$ NMR time scale at $303 \mathrm{~K}$. Thus, it appears that molecules of compound $\mathbf{2}$ can undergo rearrangement via the rotation of $\mathrm{Cu}(\beta$-diketonate) moiety about the $\mathrm{Cu}$-olefin bond.

4. Chemical Vapor Deposition of Copper from (hfac)Cu(7-t-BuO-NBD). Chemical vapor deposition experiments in the hot-wall system were carried out with (hfac) $\mathrm{Cu}(7-t$-BuO-NBD) as precursor over the deposition temperature range $140-260^{\circ} \mathrm{C}$. The precursor was sublimed at $80^{\circ} \mathrm{C}$ under reduced pressure $(0.1$ Torr) and was transferred through the deposition zone. Durations of deposition were $60-90 \mathrm{~min}$, and the film thicknesses were 1.5-3.5 $\mu \mathrm{m}$. Continuous copper films with a metallic color and resistivities less than $6 \mu \Omega \cdot \mathrm{cm}$ were deposited on both $\mathrm{SiO}_{2}$ - and Pt-coated substrates at deposition temperatures above $200{ }^{\circ} \mathrm{C}$. Hence, the

(18) Baum, T. H.; Larson, C. E.; May, G. J. Organomet. Chem. 1993, 425,189 .

(19) (a) Salomon, R. G.; Kochi, J. K. J. Am. Chem. Soc. 1973, 95, 1889. (b) Salomon, R. G.; Kochi, J. K. J. Organomet. Chem. 1974, 64, 135 .

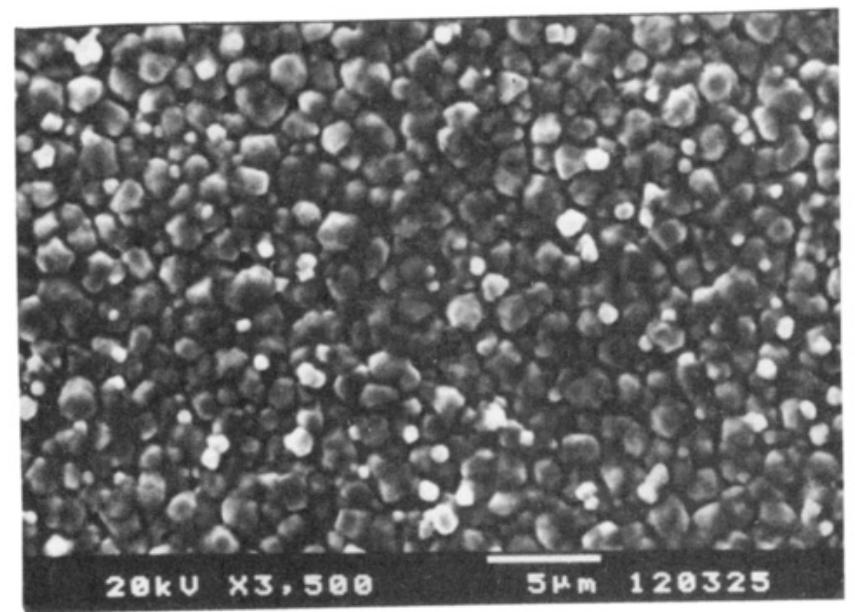

(a) $230^{\circ} \mathrm{C}$

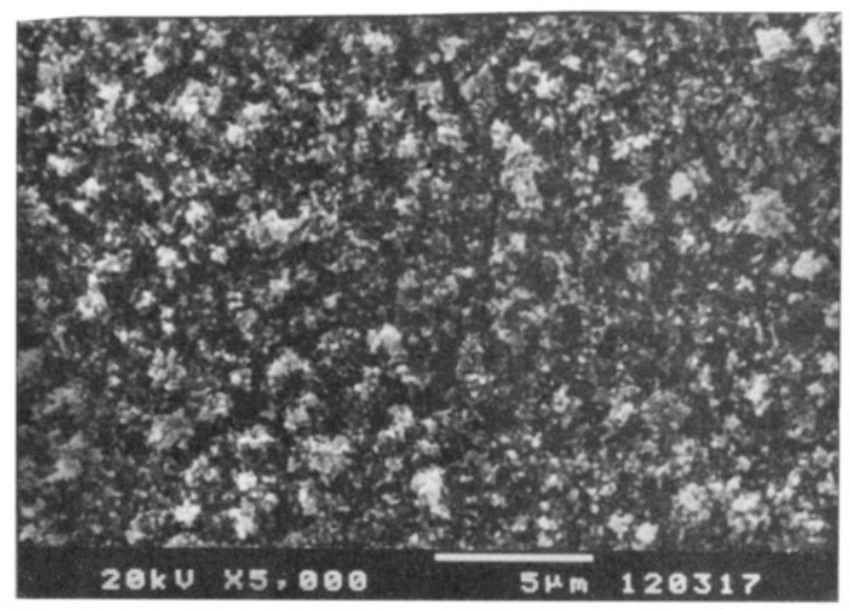

(b) $140^{\circ} \mathrm{C}$

Figure 6. Scanning electron micrographs of copper films deposited from (hfac)Cu(7-t-BuO-NBD) at (a) $230{ }^{\circ} \mathrm{C}$ and (b) $140{ }^{\circ} \mathrm{C}$.

$\mathrm{Cu}(\mathrm{I})$ precursor may undergo disproportionation to form $\mathrm{Cu}$ metal and greenish-blue $\mathrm{Cu}(\mathrm{hfac})_{2}$ which was observed on the cool end of the CVD reactor. The same mechanism was proposed for the CVD reactions with other $\beta$-diketonate complexes of copper(I) as precursors by Hampden-Smith. ${ }^{6-8}$ All copper films had good adhesion according to the Scotch tape test. The surface morphology of the films was determined with SEM; a typical example deposited on a Pt-coated Si substrate at $230{ }^{\circ} \mathrm{C}$ appears in Figure 6a. The films deposited at temperatures above $200^{\circ} \mathrm{C}$ consist of continuous grains similar to those in previous reports. ${ }^{6-8,10 \mathrm{c}}$ The grain size increases with increasing deposition temperature. $\mathrm{X}$ ray diffraction analyses of these films show the existence of crystalline material. A diffraction pattern of a film deposited on a $\mathrm{SiO}_{2}$-coated substrate at $200{ }^{\circ} \mathrm{C}$ (Figure 7) corresponds to cubic copper.

Films deposited at the temperatures below $170{ }^{\circ} \mathrm{C}$ have a dark red color that is distinct from that of films deposited at temperatures $>200^{\circ} \mathrm{C}$. It is possible that these films contain significant amount of impurities that result in larger resistivities (>1000 $\mu \Omega \cdot \mathrm{cm}$ ). These films have a morphology (Figure 6b) apparently distinct from those deposited at higher temperatures, which indicates 


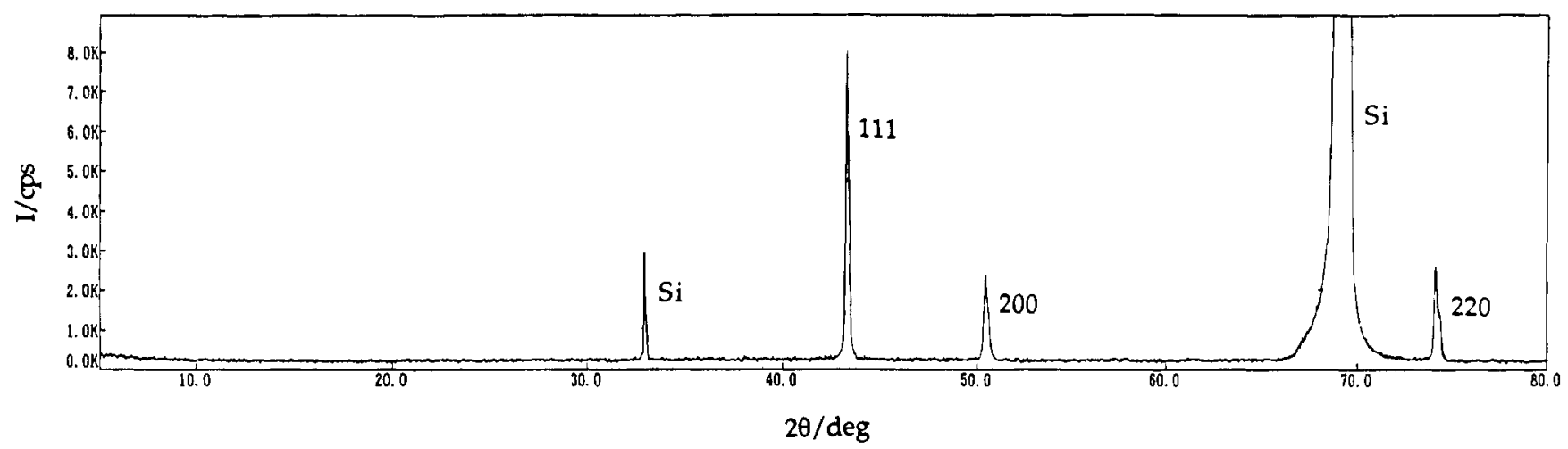

Figure 7. XRD pattern of a film deposited on a $\mathrm{SiO}_{2}$-coated substrate at $200{ }^{\circ} \mathrm{C}$.

a different composition. Although all title compounds are volatile, compounds 2-6 exhibited insufficient thermal stability at sublimation temperatures to allow CVD experiments.

In conclusion, we prepared new copper(I) complexes of the general formula ( $\beta$-diketonate) $\mathrm{Cu}(7-t$-BuO-NBD). All compounds were characterized by analytical and spectral methods. Structural properties of three compounds were determined by single-crystal X-ray diffraction analyses. ${ }^{1} \mathrm{H}$ NMR spectra of all compounds in solution at varied temperatures indicated rapid rearrangements of these flexible molecules. CVD experiments using (hfac) $\mathrm{Cu}(7-t-\mathrm{BuO}-\mathrm{NBD})$ as precursor demonstrated deposition of pure copper films at temperatures above $200^{\circ} \mathrm{C}$.
Acknowledgment. We thank Professor S. P. Wang of Department of Chemistry, National Cheng Kung University, for discussion of the NMR data, Dr. S.-G. Shyu of Institute of Chemistry, Academia Sinica, for obtaining SEM data, and the National Science Council of the Republic of China (NSC 82-0208-M-194-025) for support of this work.

Supplementary Material Available: Crystallographic data for compounds 1,4 , and 5 , including tables of crystal data, atomic coordinates, bond distances and angles, anisotropic thermal parameters ( 30 pages). Ordering information is given on any current masthead page.

OM940886D 